\title{
At last: classification of human mammary cells elucidates breast cancer origins
}

\author{
Robert D. Cardiff and Alexander D. Borowsky
}

Center for Comparative Medicine and Department of Pathology and Laboratory Medicine, Davis School of Medicine, UCD, Davis, California, USA.

\begin{abstract}
Current breast cancer classification systems are based on molecular evaluation of tumor receptor status and do not account for distinct morphological phenotypes. In other types of cancer, taxonomy based on normal cell phenotypes has been extremely useful for diagnosis and treatment strategies. In this issue of the JCI, Santagata and colleagues developed a breast cancer classification scheme based on characterization of healthy mammary cells. Reclassification of breast cancer cells and breast cancer tissue microarrays with this system correlated with prognosis better than the standard receptor status designation. This scheme provides a major advance toward our understanding of the origin of the cells in the breast and breast cancers.
\end{abstract}

\section{All tumors are not created equal}

Considering all tumors of the breast to be equal in origin, diverging only based on clonal evolution, is akin to lumping all tumors of the alimentary canal together. Such gross characterization would ignore distinct differences of cell and tissue types: just as in the gastrointestinal tract, where the squamous epithelium of the esophagus transitions to the acid- and mucousproducing epithelia of the stomach, then to the absorptive villi of the small intestine and the gland crypts of the colon, the breast is lined by specialized cells and stroma that transition from the nipple ducts to the lactiferous sinuses, from the primary ducts to the branched ducts and terminal ducts, into individual terminal ductal lobular units (TDLUs). Within each of these sites, there is a mixed distribution of cells with specialized functions. Arguably, each site and each cell or its progenitor might have higher or lower susceptibility to cancer, with differing sensitivity or resistance to environmental cancer stimuli; therefore, these distinct regions might give rise to cancers with quite different clinical behaviors.

In fact, some of the most useful cancer classifications are based on an adequate understanding of the normal cell types within a given tissue or organ. The prime examples and models for such classification are the hematopoietic malignancies. In this issue of the JCI, Santagata and colleagues

Conflict of interest: The authors have declared that no conflict of interest exists.

Citation for this article: J Clin Invest. 2014; 124(2):478-480. doi:10.1172/JCI73910. have developed a breast cancer classification system based on phenotypes associated with normal mammary tissue (1).

\section{Breast cancer diagnosis and classification: a brief history}

From the early days of microscopic pathology, the pathology and natural history of breast cancer have been controversial (2). Waldeyer gets credit for the first description of a breast cancer in direct continuity with normal mammary epithelium $(2,3)$. Although his mentor Virchow favored the blastema hypothesis, which suggests that cancer cells originate from budding elements between normal tissues, Waldeyer's concept that breast cancer originates from breast epithelium has survived to become universally accepted. For many years, Halstead's concept that breast cancer metastasizes through centripetal spread led to increasingly radical mastectomies; however, dissenters such as Greenough (4) and Macdonald (5) contested his concepts of natural history and proposed that some tumors were more aggressive than others at initiation (6). Foote and Stewart began serious morphological subclassification of breast cancers with the descriptive misnomer lobular carcinoma (7); the early history of carcinogenesis (2) and the history of the conflicting views of breast cancer $(6,8)$ have previously been discussed in detail.

Evidence-based pathology dating back to the 1970s and 1980s resulted in lumping a heterogeneous array of tumor morphologies, which constitute the majority of breast cancers, under the heading of invasive ductal carcinoma, although this classification would be better named invasive mammary carcinoma of no special type. This era culminated in the current WHO morphological breast cancer classification based on histological pattern (9). Newer high-throughput molecular analyses of breast cancers have ignored morphological classifications and have simplified breast cancer classifications as being luminal or basal. These molecular classifications imply that there is no practical reason to separate these morphologies, and classification of special types of breast cancer with distinct morphological properties was regarded as a list of heterogeneous minority phenotypes without much value (1).

Unique morphological types have largely been discarded in current clinical practice. Instead, breast cancers are assayed for clinically useful targets based on the presence or absence of the magic targetable twosome, estrogen receptor (ER) and human epidermal growth factor receptor 2 (HER2). Tumors that do not contain either of the magic treatable receptors are categorized as triple-negative breast cancer (TNBC), encompassing a diverse group of tumors without druggable targets $(10,11)$. An unfortunate overlap of TNBC cancers with the genomic subset of basal-like cancers has cemented into place the concept that breast cancers are either luminal or basal, based on molecular criteria. Furthermore, simplifications about the nature and distribution of normal cell markers (such as cytokeratin 5, which is erroneously believed to be only in basal cells) continue to confuse the literature $(12,13)$.

\section{Characterization of mammary cell types provides better breast cancer diagnostics}

In response to the need to be translationally relevant (i.e., fundable), basic breast cancer research tumor classifications have focused primarily on identifying cells with stem-like and progenitor-like properties. While these properties are hot topics, they do not define actual differentiation states of mature cells in the breast beyond con- 
ventional designations of basal verses luminal epithelial, and ductal verses lobular. Unfortunately, current descriptions of the human breast ductal/lobular tree taxonomy are oversimplified and often presented as possible or conjectured without empirical observations. In our view, the work to date on breast stem, progenitor, or "transit-amplifying" cells is limited by an incomplete understanding of the hierarchical taxonomy of mammary cells. Without this basis, it is not surprising that markers identifying cells with stem-like behavior in one context do not work for identifying cancer-driving cells in another. We anticipate, as proposed by Santagata et al. (1), that a hierarchy of undifferentiated, lineage-restricted, and lineage-committed stem and progenitor cells give rise to the array of luminal cell types outlined by their analyses, as well as a similarly complex array of basally oriented lining cells.

The paper by Santagata et al. represents a major advance toward a realistic, biologically based taxonomy of mammary cells and illustrates the utility of this taxonomy in evaluation of cancers of the breast (1). The authors painstakingly analyzed the normal lobules from 36 "normal" mammoplasties. Their studies led to a more comprehensive understanding of the distribution of antigens expressed in normal human mammary epithelium and to an evidence-based classification scheme. Immunofluorescence and immunohistochemistry were used to define cell groups based on hormone receptors (HRs; namely, ER, androgen receptor [AR; not progesterone receptor], and vitamin D receptor [VDR]), intermediate filaments (keratin 5 [K5], K6, K7, K14, and $\mathrm{K} 18$ ), the proliferation marker Ki67, and myoepithelial markers (CD10, p63, and SMA). Evaluation of these markers in luminal cells from normal human breast TDLUs could separate eleven luminal subtypes and two subsets within the myoepithelial compartment (1). Based on these insights, the authors proposed an ontology scheme based primarily on the number of HRs present (designated HR0-HR3), and a secondary categorical separation of HR0 tumors (which lack all three HRs) based on the presence or absence of K5. At the HR2 level, cells could be distinguished by the HR combination (i.e., which two of the three HRs were detected) as well as K5 status. Interestingly, proliferating cells $\left(\mathrm{Ki} 7^{+}\right)$ were HR0 and produced K18. Furthermore, HER2 was not expressed in normal TDLUs.

Table 1

HRO-HR3 reclassification of breast tumors

\begin{tabular}{lcccc}
\hline & ER $^{+}$ & HER2+ & TNBC & Total \\
HR0 & $0.0 \%$ & $5.1 \%$ & $36.8 \%$ & $6.1 \%$ \\
HR1 & $1.5 \%$ & $22.0 \%$ & $44.6 \%$ & $10.8 \%$ \\
HR2 & $23.4 \%$ & $43.5 \%$ & $18.6 \%$ & $25.0 \%$ \\
HR3 & $75.1 \%$ & $29.4 \%$ & $0.0 \%$ & $58.1 \%$ \\
\hline
\end{tabular}

Frequency of HRO, HR1, HR2, and HR3 tumors in the clinical categories ER+ ${ }^{+}, \mathrm{HER} 2^{+}$, and TNBC. Table adapted from Journal of Clinical Investigation (1).

These extensive observations will serve as a foundation for future understanding of human breast cancer.

Despite these great advances in breast cancer classification, the study by Santagata and colleagues (1) is primarily based on single-time-point analysis of clinically derived excisions and formalin-fixed, paraffin-embedded sections of breast tissue. Based on this snapshot analysis, one is left to wonder whether all luminal cells go through a defined sequence of immunnophenotypes that diverge at specific branch points, similar to hematopoietic differentiation, or are immunophenotypes that prove transient as each cell reacts and adapts to its microenvironment. For example, $\mathrm{Ki}^{+} 7^{+}$cells that are characterized as HR0 and $\mathrm{K} 18^{+}$must produce daughter cells belonging to all the other lineages if the mammary gland is to proliferate, or if breast tumors are to maintain their HRdependent phenotype. Lineage definition through experimental manipulation will be almost impossible in humans; therefore, mammary cell lineage tracing will have to be examined using mammalian models, such as the mouse.

Moving forward on the assumption that their new taxonomy defines the cell of origin, Santagata et al. reclassified several collections of breast cancers and breast cancer tissue microarrays (TMAs). Excitingly, their classification scheme organized human breast cancers into categories that were relevant to prognosis (1). Comparison of the reclassified groups with the current categories revealed interesting mixtures of HR0-HR3 tumors within $\mathrm{ER}^{+}$and HER2 ${ }^{+}$ groupings (Table 1), which may explain why therapeutic strategies based on current standards of classification have failed.

In addition to using their taxonomy system to characterize breast cancer cells, Santagata and colleagues applied their classification to the collection of $50 \mathrm{NIH}$ human breast cancer cell lines. Interestingly, nine of the cell lines contained none of the markers found to be associated with breast cancer (1). The cell lines tested included MDA-MB-231, SUM-159PT, MDAMB-157, MDA-MB-436, HBL100, BT549, SUM131, 5M02, MDA-MB-435, and HS578T, which were originally thought to be representative of human breast cancers, but are no longer considered by most experts to be breast cancer cells. The fact that the HR designation also indicated that these cell lines are not representative of human breast cancer is encouraging. Scientists that use mouse models of breast cancer should be delighted to learn that the basal cell designation can be discarded, because human breast cancers did not contain this specific cell population.

The discontent between complex molecular profiling and simple morphological approaches can be seen in a plethora of other breast cancer-associated classification systems, which also rely on more recent molecular and immunohistochemical classifications to claim utilitarian value for their particular schemata. The shortcoming of all these taxonomies of human breast cancer has been a lack of biological basis, which is now provided by Santagata and colleagues (1). The authors cautiously point out that their work is a starting point and not yet a complete description of TDLU taxonomy. Meanwhile, they have provided ample clinically based data to justify the use of such a classification system. The question of whether the HRO-HR3 designation improves our understanding and treatment of breast cancer or simply adds to our current confusion remains to be answered. Although the present study is complex and provides valid clinical justifications for use of the HR0-HR3 classification scheme, the most important aspect of this report is that it provides a biological basis for testing various clinical and scientific hypotheses regarding the origins of breast cancers. 


\section{Acknowledgments}

The authors' work is supported by NCI grants U01 CA141541 and U01 CA141582 and by Athena Breast Health Network through the University of California Office of the President.

Address correspondence to: Robert D. Cardiff, Center for Comparative Medicine, UCD, Road 98 and Hutchison Drive, Davis, California 95616, USA. Phone: 530.752.2726; Fax: 530.752.7914; E-mail: rdcardiff@ucdavis.edu.
1. Santagata S, et al. Taxonomy of breast cancer based on normal cell phenotype predicts outcome. J Clin Invest. 2014;124(2):859-870

2. Rather LJ. The Genesis of Cancer: A Study in the History of Ideas. Baltimore, Maryland, USA: Johns Hopkins University Press; 1978.

3. Waldeyer HWG. Die Entwickelung der Carcinome. Virch Arch Pathol Anat. 1867;41:470-523.

4. Greenough RB, Taylor GW. Cancer of the breast, end results. N Engl J Med. 1934;210:831-835.

5. Macdonald I. Biological predeterminism in human cancer. Surg Gynecol Obstet. 1951;92(4):443-452.

6. Lerner BH. Great expectations: historical perspectives on genetic breast cancer testing. Am J Public Health. 1999;89(6):938-944.

7. Foote FW, Stewart FW. Lobular carcinoma in situ: a rare form of mammary carcinoma. Am J Surg Pathol. 1941;17(4):491-496.
8. Lerner BH. The Breast Cancer Wars: Hope, Fear, And The Pursuit Of A Cure In Twentieth-Century America. New York, New York, USA: Oxford University Press; 2001. 9. Tavassoli FA, Devilee P. International Agency for Research on Cancer., World Health Organization: Pathology and genetics of tumours of the breast and female genital organs. Lyon, France: IAPS Press; 2003.

10. Rakha EA, et al. Are triple-negative tumours and basal-like breast cancer synonymous? Breast Cancer Res. 2007;9(6):404.

11. Reis-Filho JS, Tutt AN. Triple negative tumours: a critical review. Histopathology. 2008;52(1):108-118.

12. Gusterson B. Do 'basal-like' breast cancers really exist? Nat Rev Cancer. 2009;9(2):128-134.

13. Moinfar F. Is 'basal-like' carcinoma of the breast a distinct clinicopathological entity? A critical review with cautionary notes. Pathobiology. 2008; 75(2):119-131.

\title{
An unexpected role for platelets in blocking Th17 differentiation
}

\author{
Ronjon Chakraverty
}

\begin{abstract}
Transplantation Immunology Group, Cancer Institute and Institute for Immunity and Transplantation, University College London, London, United Kingdom.
\end{abstract}

\begin{abstract}
It is well known that platelets interact with cells of the innate immune system to promote tissue repair. In contrast, it is less clear whether these links extend to cells of the adaptive immune system, such as $T$ cells. In this issue of the JCI, Morrell and colleagues provide compelling evidence that platelets are required to limit $\mathrm{CD} 4^{+} \mathrm{Th} 17$ differentiation through the actions of the chemokine platelet factor 4 (PF4). Absence of PF4 in the host leads to exaggerated Th17 differentiation after transplantation and rapid graft rejection. The authors' findings argue that platelets are not bit part players, but rather fully fledged, critical members of the adaptive immune system.
\end{abstract}

\section{Platelets as a bridge between coagulation and innate immunity}

In addition to their role in the hemostatic response to vascular injury, platelets provide an essential link to the innate immune system, enabling wound repair and reestablishing tissue homeostasis (1). Platelets are well positioned to act as a conduit between the coagulation and immune systems by providing a physical scaffold for cell interactions and delivering a large repertoire of immune mediators through exocytosis from storage granules or extrusion of membrane- and cytoplasm-containing microparticles (1). One of the most abundant platelet-derived immune molecules is the chemokine platelet factor 4 (PF4; also known as CXCL4). Upon platelet activation, $\mathrm{PF} 4$ is rapidly released from cytosolic $\alpha$ granules, leading to a huge increase in

Conflict of interest: The author has declared that no conflict of interest exists.

Citation for this article: J Clin Invest. 2014;

124(2):480-482. doi:10.1172/JCI74231. local concentration (2). The functions of PF4 are complex, generating both pro- and anticoagulant actions (3) and differentially affecting multiple cell types, including megakaryocytes, endothelial cells, and innate immune cells (2-4). For example, in response to endothelial injury, plateletderived PF4 is crucial for monocyte recruitment, survival promotion, and proinflammatory macrophage differentiation (5). PF4 also binds other chemokines, such as CXCL8 (6) and CCL5 (5), to generate heteromers with distinct activities. Together, the diverse actions of PF4 pose an enormous challenge to investigators attempting to understand its physiological functions.

The interaction between platelets and a damaged endothelium is a common feature of many disorders in humans. In the chronic inflammatory lesions associated with atherosclerosis, PF4 is critical for macrophage infiltration and disease progression (5). Endothelial injury may also occur in transplanted organs, either as a result of the effects of ischemia and reperfusion or as the result of antibody and cellular responses within the host (7). Furthermore, platelets have been proposed to be important mediators of the initial host response to grafted tissue, but it is not clear how platelets contribute to the ensuing adaptive immune response.

\section{Loss of PF4 accelerates graft rejection by enhancing Th17}

In this issue of the JCI, Shi, Morrell, and colleagues examined how platelets and platelet-derived PF4 affect anti-donor T cell responses after transplantation (8). Based on the broad premise that platelets and PF4 induce a proinflammatory response, the authors hypothesized that deletion of Pf4 in host mice would protect MHC class II-mismatched cardiac allografts from rejection. In this murine model of cardiac transplant, graft rejection is usually mediated by classical Th1-type responses, where $\mathrm{CD}^{+} \mathrm{T}$ cell effectors produce cytokines such as IFN- $\gamma$, IL-2, and GM-CSF (9). Although Th1 differentiation was disabled in $\mathrm{Pf}^{-/-}$hosts, Morrell and colleagues surprisingly found that grafts in these mice were rejected at an earlier time point compared with control animals (8). Histological examination of rejected grafts from $\mathrm{Pf}^{-/-}$mice revealed an atypical vasculopathy, with perivascular aggregates composed of $\mathrm{T}$ cells and dense infiltrates of neutrophils. Similar neutrophil recruitment after transplantation has been previously observed in Tbx $21^{-/-}$mice, 